\title{
Purine Metabolites and Lactate as Parameters of Hypoxia in the Newborn Infant
}

\author{
VINETA RUTH AND KARI O. RAIVIO(19) \\ Children's Hospital and Departments of Obstetrics and Gynecology, University of Helsinki, Finland
}

\begin{abstract}
Summary
In order to evaluate possible biochemical parameters of tissue hypoxia, arterial samples from 29 sick neonates were taken at 6$h$ intervals for lactate and blood gas analyses, as well as assays of purine metabolites in plasma. The state of the infant at each sampling was arbitrarily classified as severe, moderate, or mild on the basis of $\mathrm{Po}_{2}, \mathrm{pH}, \mathrm{FiO}_{2}$, and transcutaneous $\mathrm{Po}_{2}$. Of the total of 289 samples, 72 were in the mild, 178 in the moderate, and 39 in the severe category. Elevated values for hypoxanthine $(>36 \mu \mathrm{mol} / \mathrm{L})$, xanthine $(>11 \mu \mathrm{mol} / \mathrm{L})$, and lactate $(>3.6 \mathrm{mmol} /$ L) were defined as $>2$ SD above the mean of infants with slight or no hypoxia. Hypoxanthine values were elevated in $7 \%$ of mild and in $13 \%$ of severe cases whereas the corresponding incidences for elevated xanthine values were 1 and $8 \%$, and for elevated lactate values 7 and $39 \%$. Conversely, when hypoxanthine was elevated $(n=21)$, mild hypoxia was present in $24 \%$ and severe in the same percentage of cases whereas the corresponding incidences for elevated xanthine $(n=7)$ were 14 and $43 \%$ and for lactic acid $(n=41) 12$ and $37 \%$. No consistent differences in plasma uric acid levels were noted between the mild and severe cases of hypoxia. We conclude that neither purine metabolites nor lactic acid concentrations in intermittent samples of arterial blood are reliable indicators of tissue oxygenation in the newborn.
\end{abstract}

\section{Abbreviations}

HPLC, high performance liquid chromatography IMP, inosinate

RDS, respiratory distress syndrome

tcPo $\mathrm{PO}_{2}$, transcutaneous $\mathrm{PO}_{2}$

Disturbances in the oxygen supply at the cellular level result in metabolic changes, some of which reflect the severity of the oxygen deficiency. One of these changes is anaerobic lactate formation, resulting in metabolic acidosis which is used in clinical work as a parameter of hypoxia. Another is catabolism of high energy phosphates, especially ATP. Measurement of breakdown products of purine nucleotides in plasma has been proposed as a useful biochemical parameter of perinatal hypoxia. Preliminary clinical studies $(2,9,13)$ and animal experiments $(14,17)$ have suggested that hypoxanthine might be a suitable intermediate to measure, although uric acid is also known to be elevated in neonates with respiratory problems (8). Evaluation of hypoxanthine in this respect has been limited so far to cord blood samples $(6,9,13)$ and to arterial samples from a small number of sick neonates during the first hours of life $(2,12)$. Poor correlation between cord plasma hypoxanthine concentration and fetal asphyxia has also been reported (6). In adults, lactate and electrocardiography have been reported to be superior to hypoxanthine as indices of tissue hypoxia (18).

The purpose of this study was to assess whether hypoxanthine, xanthine, uric acid, or lactate concentrations are correlated with the prevailing oxygenation state of the newborn, classified by clinical criteria, $\mathrm{PaO}_{2}$ and acid-base measurements in arterial blood. For this purpose, we studied infants in intensive care, who had an umbilical arterial catheter in place.

\section{PATIENTS AND METHODS}

The study was conducted in the neonatal intensive care unit of the Children's Hospital, University of Helsinki, during a 6mo period. Infants who weighed over $1000 \mathrm{~g}$ and had an umbilical arterial catheter for monitoring oxygen therapy for 2 or more days were included. Their main clinical characteristics are summarized in Table 1. Patients who died before completion of the study were excluded.

The birthweight of all 29 infants was appropriate for gestational age. All needed ventilatory assistance, although not necessarily during the 48-h study period, and this was carried out according to generally accepted principles (4). In addition to intermittent arterial sampling, tc $\mathrm{PO}_{2}$ was monitored in each patient. Four of the infants died in the neonatal period after the study, two of congenital heart disease and two of septicemia.

During the actual study period of at least $48 \mathrm{~h}$, arterial blood samples were drawn into heparinized syringes at 6 -h intervals. An aliquot was precipitated with perchloric acid for lactate determination. Another aliquot was used for acid-base and $\mathrm{PO}_{2}$ analysis. The rest of the sample was centrifuged and plasma frozen for purine analysis. Sample preparation was completed as rapidly as clinical routine allowed, in all cases within $1 \mathrm{~h}$.

Table 1. Characteristics of patients $(n=29)$

\begin{tabular}{lc}
\hline \multicolumn{1}{c}{ Characteristic } & No. of Patients \\
\hline Birthweight $(\mathrm{g})$ & 7 \\
$1000-1500$ & 10 \\
$1501-2500$ & 12 \\
$>2500$ & \\
Gestational age $(\mathrm{wk})$ & 10 \\
$<32$ & 11 \\
$32-36$ & 6 \\
$37-41$ & 2 \\
$\geq 42$ & 16 \\
Diagnoses & 5 \\
Respiratory distress syndrome & 4 \\
Septicemia & 3 \\
Persistent fetal circulation & 1 \\
Congenital heart disease & \\
Asphyxia & 14 \\
Age at onset of study (h) & 6 \\
$<12$ & 6 \\
12-24 & 3 \\
$24-48$ & \\
$>48$ &
\end{tabular}


At the time of each arterial blood sample, the infant was classified as having either mild, moderate, or severe hypoxia. The classification was based on arterial $\mathrm{pH}$ and $\mathrm{PaO}_{2}$. Transcutaneous $\mathrm{PO}_{2}$ recordings over the $6 \mathrm{~h}$ preceding the sample were available in 19 patients. These were used as additional criteria to estimate past hypoxia. For inclusion into the mild group, a pH of 7.30 or higher and $\mathrm{a} \mathrm{PaO}_{2}$ of $50 \mathrm{~mm} \mathrm{Hg}$ or higher at an $\mathrm{FiO}_{2}$ of 0.40 or lower were required. In addition, $\mathrm{tcPO}_{2}$ levels of less than $40 \mathrm{~mm} \mathrm{Hg}$ were permitted for a maximum of $30 \mathrm{~min}$ and levels of less than $25 \mathrm{~mm} \mathrm{Hg}$ for a maximum of 5 min during the $6 \mathrm{~h}$ preceding the sample. For inclusion into the severe group, a pH value below 7.20 (metabolic acidosis) and/or a $\mathrm{PaO}_{2}$ value below $40 \mathrm{~mm} \mathrm{Hg}$ was required. $\mathrm{FiO}_{2}$ at the time of sampling was 0.80 or higher in $62 \%$ of these cases, and below 0.50 in $15 \%$. Also, $\mathrm{tcPO}_{2}$ levels below $40 \mathrm{~mm} \mathrm{Hg}$ for over $120 \mathrm{~min}$ or levels below $25 \mathrm{~mm} \mathrm{Hg}$ for over 15 min during the preceding $6 \mathrm{~h}$ and at the time of sampling qualified for the severe group. If the $\mathrm{pH}$ and $\mathrm{PO}_{2}$ values fell between the criteria for mild and severe hypoxia, the sample was assigned to the moderate group.

Although our classification is arbitrary, we consider that the mild and severe categories represent clearly different situations with respect to oxygenation although both may overlap with the intermediate category. For this reason, results will be presented mainly for these two groups and comparisons made between them.

Ethical considerations precluded arterial blood sampling from normal newborns. Reference values for hypoxanthine and lactate were, therefore, derived from a subgroup of mild hypoxia, with stricter criteria for "normalcy" (pH 7.35 or higher and $\mathrm{PaO}_{2} 50$ $\mathrm{mm} \mathrm{Hg}$ or higher at an $\mathrm{FiO}_{2}$ of 0.25 or lower). These 12 samples were usually obtained just before removal of the catheter. Mean \pm SD for hypoxanthine in this group was $22 \pm 7 \mu \mathrm{mol} / \mathrm{L}$ and for lactate $2.4 \pm 0.8 \mathrm{mmol} / \mathrm{L}$. Elevated values for these parameters were defined as those exceeding the mean of this subgroup by more than $2 \mathrm{SD}$. Half of the xanthine values in this group were zero and the range was up to $10 \mu \mathrm{mol} / \mathrm{L}$.

The $\mathrm{pH}$ and $\mathrm{PO}_{2}$ were measured with a Radiometer blood gas analyzer. Lactate was assayed enzymatically using a commercial reagent kit (Boehringer Mannheim, Germany). For measurement of purine nucleosides and bases, plasma was deproteinized with perchloric acid, and an aliquot of the neutralized supernatant was analyzed with a HPLC system (Waters Associates) using a $\mu$ Bondapak $\mathrm{C} 18$ reverse phase column (1). Of the compounds separated and quantitated, data are presented for only hypoxanthine, xanthine, and uric acid. Uric acid was measured spectrophotometrically (Urica-Quant reagent kit, Boehringer Mannheim, Germany), and the results agreed with the HPLC analyses to within $10 \%$.

\section{RESULTS}

From the 29 infants, a total of 289 samples were obtained. By our definition, 72 of these were drawn while the infant had mild hypoxia, 178 were in the moderate, and 39 in the severe category.

Purine metabolites. The hypoxanthine concentrations measured in the mild and severe groups are shown in Figure 1. With a single exception in the severe group, the ranges are almost identical, as are the means for both groups $(20.9 \mu \mathrm{mol} / \mathrm{L}$ in the mild and $23.3 \mu \mathrm{mol} / \mathrm{L}$ in the severe). The proportion of values exceeding the upper limit of "normal" $(36 \mu \mathrm{mol} / \mathrm{L})$ was $7 \%$ in the mild and $13 \%$ in the severe group.

Out of the total of 289 plasma samples, 21 had an elevated hypoxanthine concentration, i.e., in excess of $36 \mu \mathrm{mol} / \mathrm{L}$. Five of these were measured in mildly and five in severely hypoxic infants, and 11 belonged to the moderate group.

The molar concentrations of xanthine in plasma were lower than those of hypoxanthine (Fig. 1), but again there was extensive overlap between the values of the mild and severe groups. The means were $2.8 \mu \mathrm{mol} / \mathrm{L}$ and $4.9 \mu \mathrm{mol} / \mathrm{L}$, respectively and only one of the mild and three of the severe group exceeded the "normal" upper limit of $11.2 \mu \mathrm{mol} / \mathrm{L}$.

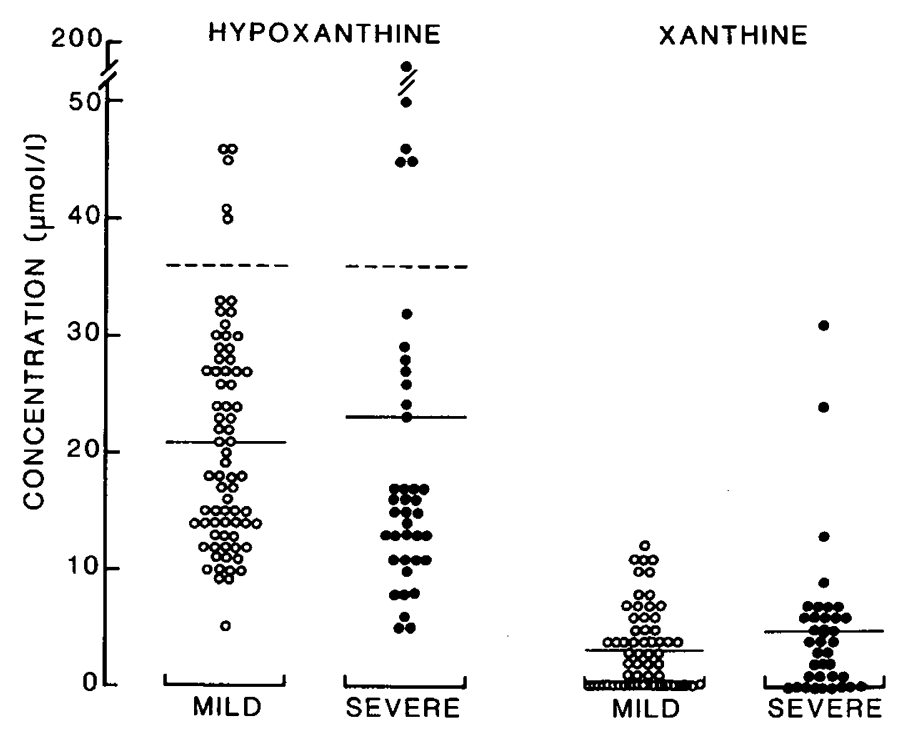

Fig. 1. Arterial plasma hypoxanthine and xanthine concentrations in newborn infants with mild or severe hypoxia. Solid lines indicate means for each group and broken lines represent means + SD of plasma hypoxanthine in a group of infants with slight or no hypoxia (see text).

As previously shown for normal and hypoxic neonates (8), plasma uric acid concentrations increased after birth and started to decrease on the second or third day of life. This pattern was followed, with five exceptions, by all the infants studied whereas neither hypoxanthine nor xanthine concentrations showed any age-dependence. "Normal" reference values could not be derived for uric acid because of too few samples in each age group; however, there were no consistent differences in plasma uric acid levels between the mild and severe cases of hypoxia within the age range studied (Fig. 2). The variation within each group was extensive but almost all values, even in mild hypoxia, were higher than the mean values previously measured in capillary blood of a group of normal newborn infants (Fig. 2).

Lactic acid. The results of lactic acid measurements, like those of purine metabolites, showed marked overlap of values in the mild and severe categories of hypoxia. The mean in the severe group $(3.8 \mathrm{mmol} / \mathrm{L})$ was higher than in the mild $(2.3 \mathrm{mmol} / \mathrm{L})$ (Fig. 3), but the difference was not statistically significant (MannWhitney test). A larger proportion of samples in the severe group (39\%) had values in excess of the upper limit of "normal" (3.6 $\mathrm{mmol} / \mathrm{L}$ ) whereas the proportion in the mild group was only $7 \%$. If all the elevated lactate values $(n=41)$ are considered, 15 of them were encountered in the severely hypoxic infants and five in the mild category. It is likely that our classification, which was partly based on $\mathrm{pH}$ values, accounts for the larger incidence of elevated lactate values in the severe group.

Correlations between hypoxia parameters. When the findings in the total material were subjected to linear regression analysis, no correlation could be established between the concentrations of any of the purine metabolites, lactic acid, $\mathrm{PaO}_{2}$, or $\mathrm{pH}$. Within the severely hypoxic group, hypoxanthine and xanthine were highly significantly $(r=0.831, P<0.001)$, and hypoxanthine and lactate almost significantly $(r=0.312, P<0.05)$ correlated, but all the other cross-correlations were non-significant.

Of the most elevated values encountered, the highest hypoxanthine concentration $(198 \mu \mathrm{mol} / \mathrm{L})$ was measured in an infant with severe RDS immediately after an episode of pneumothorax and $30 \mathrm{~min}$ of resuscitation, i.e., severe hypoxia. The same sample had an elevated xanthine $(31 \mu \mathrm{mol} / \mathrm{L})$ and lactic acid $(9.2 \mathrm{mmol} / \mathrm{L})$ concentration, a pH of 7.17 , but normal $\mathrm{PaO}_{2}(63$ $\mathrm{mm} \mathrm{Hg}$ at $\left.1.0 \mathrm{FiO}_{2}\right)$ and uric acid $(490 \mu \mathrm{mol} / \mathrm{L}$ at $15 \mathrm{~h}$ of age). Six hours later, hypoxanthine had decreased to $53 \mu \mathrm{mol} / \mathrm{L}$ and xanthine to $24 \mu \mathrm{mol} / \mathrm{L}$ whereas uric acid had sharply increased to $765 \mu \mathrm{mol} / \mathrm{L}$ and lactic acid to $12.1 \mathrm{mmol} / \mathrm{L}$. Very high uric acid concentrations were measured twice in another infant with 


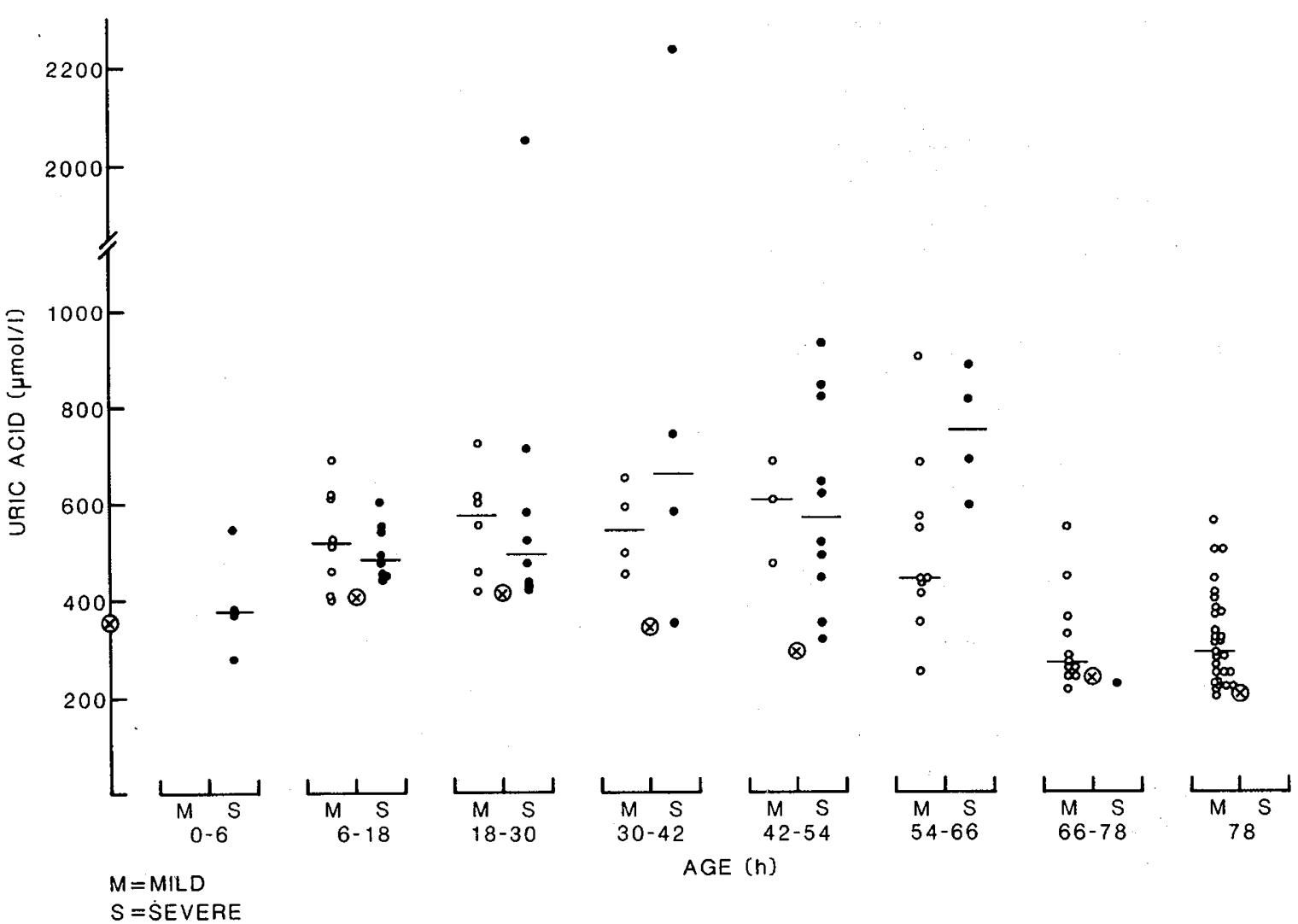

Fig. 2. Arterial plasma uric acid concentrations as a function of age in newborn infants with mild $(M)$ or severe $(S)$ hypoxia. Horizontal bars indicate median values for each group. $\otimes$ indicates mean capillary plasma uric acid concentrations in a group of normal newborn infants (ref. 8 ).

severe congenital heart disease and persistent hypoxia (2050 and $2230 \mu \mathrm{mol} / \mathrm{L}$ ). Hypoxanthine (50 and $46 \mu \mathrm{mol} / \mathrm{L}$ ) and xanthine ( 24 and $13 \mu \mathrm{mol} / \mathrm{L}$ ) were simultaneously elevated, but lactic acid only slightly elevated $(3.0$ and $3.9 \mathrm{mmol} / \mathrm{L})$. pH was normal and $\mathrm{PaO}_{2}$ very low (16 and $31 \mathrm{~mm} \mathrm{Hg}$ at an $\mathrm{FiO}_{2}$ of 0.80 and 1.00 ). It appears that extreme hypoxia is associated with extreme levels of purine metabolites.

\section{DISCUSSION}

Biochemical evidence clearly shows that tissue hypoxia results in activation of glycolysis and an increase in lactate production. Yet at the level of the whole organism, blood lactate concentration has not proved a sufficiently reliable parameter of hypoxia to gain clinical acceptance. Biochemical evidence also indicates that tissue hypoxia results in decreased ATP resynthesis by oxidative phosphorylation, followed by catabolism of adenine nucleotides. Yet our findings in this study suggest that, at the level of the whole organism, oxygen deficiency correlates poorly with the plasma concentrations of the main nucleotide degradation products, hypoxanthine, xanthine, and uric acid. This result may be explained by methodologic problems, by the design of the study, or by biological factors.

For the ethical reasons mentioned, our study did not include normal newborn infants to provide reference values for the parameters measured. The mean concentration of hypoxanthine ( $22 \mu \mathrm{mol} / \mathrm{L})$ in our least hypoxic "reference" group was higher than in the cord blood of normal neonates, reported to range from $4-12 \mu \mathrm{mol} / \mathrm{L}(6,9,12)$. Methodologic problems may partly account for the difference.

We studied blood samples drawn for clinical purposes, without special arrangements for the research project; thus, it is possible that the time between drawings of the arterial sample and its centrifugation varied somewhat, depending on the routine processing of samples. When a blood sample is left standing, hypoxanthine may be released from the erythrocytes by adenine

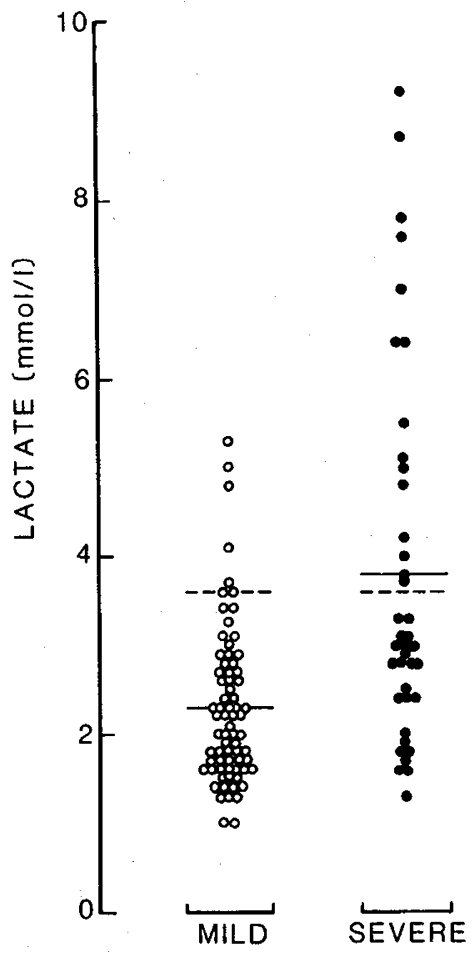

Fig. 3. Arterial blood lactate concentrations in newborn infants with mild or severe hypoxia. Solid lines indicate means for each group and broken lines represents means $+2 \mathrm{SD}$ for a group of infants with slight or no hypoxia (see text).

nucleotide breakdown $(6,10)$, but also taken up by the erythrocytes and converted into IMP (5). Likewise, adenosine or inosine, present in blood at the time of sampling, may be converted very 
rapidly into hypoxanthine (7). Because xanthine oxidase activity in blood is negligible, analogous changes in xanthine and uric acid levels are not to be expected.

Although failure to separate plasma immediately from the red cells may have contributed to the relatively high hypoxanthine concentrations in our least hypoxic "reference" cases, it does not account for lack of a difference in hypoxanthine concentrations between the mildly and the severely hypoxic samples. If a difference did in fact exist, an artifactual increase in low values and/ or a decrease in high values would have to be postulated. Because our study protocol was adjusted to clinical routine, samples were taken at predetermined intervals, which did not always coincide with fluctuations in the course of the patient. Yet our classification of the samples, although arbitrary, was based on the status of the patient at the time of sampling; thus, rapid disappearance of hypoxanthine, the half-life of elevated values being estimated at approximately $20 \mathrm{~min}(15)$, cannot account for the lack of difference between the mild and severe groups.

The possibility remains that the poor correlation of lactate and purine catabolite concentrations with clinical hypoxia is due to biological factors. Resistance to hypoxia is typical of the fetal and neonatal period. Tissues and organs vary in their resistance in terms of maintaining normal concentrations of adenine nucleotides in the face of reduced oxygen supply (16). For example, brain tissue is capable of maintaining normal adenine nucleotide concentrations at a time when oxygen deprivation has already caused several other effects, including inhibition of neurotransmitter synthesis (3). On the other hand, factors other than hypoxia may influence plasma hypoxanthine, for instance circulatory alterations $(2,11)$.

We conclude that monitoring of arterial plasma hypoxanthine or other purine metabolite concentrations is not, at the present stage of our knowledge, a clinically useful parameter of hypoxia in neonatal intensive care.

\section{REFERENCES AND NOTES}

1. Assenza, S. P. and Brown, P.: Evaluation of reversed-phase, radially compressed, flexible-walled columns for the separation of low molecular weight, UV-absorbing compounds in serum. J. Liquid Chromatogr., 3: 41 (1980).

2. Bratteby, L. E. and Swanström, S.: Hypoxanthine concentration in plasma during the first two hours after birth in normal and asphyxiated infants. Pediatr. Res., 16: 152 (1982).

3. Gibson, G. E., Pulsinelli, W., Blass, J. P., and Duffy, T. E.: Brain dysfunction in mild to moderate hypoxia. Am. J. Med., 70: 1247 (1981).

4. Goldsmith, J. P. and Karotkin, E. H.: Assisted Ventilation of the Neonate. p. 101 (Saunders, Philadelphia, PA, 1981).

5. Henderson, J. F., Zombor, G., Fraser, J. H., McCoy, E. E., Verhoef, V., and Morris, A. J.: Factors affecting inosinate synthesis and inosine triphosphate accumulation in human erythrocytes. Can. J. Biochem., 55: 359 (1977).

6. O'Connor, M. C., Harkness, R. A., Simmonds, R. J., and Hytten, F. E.: The measurement of hypoxanthine, xanthine, inosine and uridine in umbilical cord blood and foetal scalp blood samples as a measure of foetal hypoxia. Br. J. Obstet. Gynaecol., 88: 381 (1981).

7. Parks, R. E. Jr., Crabtree, G. W., Kong, C. M., Agarwal, R. P., Agarwal, K. C., and Scholar, E. M.: Incorporation of analog purine nucleosides into the formed elements of human blood: erythrocytes, platelets, and lymphocytes. Ann. N.Y. Acad. Sci., 255: 412 (1975).

8. Raivio, K. O.: Neonatal hyperuricemia. J. Pediatr., 88: 625 (1976).

9. Saugstad, O. D.: Hypoxanthine as a measurement of hypoxia. Pediatr. Res., 9 : 158 (1975).

10. Saugstad, O. D.: The determination of hypoxanthine and xanthine with a $\mathrm{pO}_{2}$ electrode. Pediatr. Res., 9: 575 (1975).

11. Saugstad, O. D., Bö, G., Östrem, T., and Aasen, A. O.: Hypoxanthine levels of plasma during hypoxemia in dogs. Eur. Surg. Res., 9: 23 (1977).

12. Swanström, S. and Bratteby, L.-E.: Hypoxanthine as a test of perinatal hypoxia as compared to lactate, base deficit, and pH. Pediatr. Res., 16: 156 (1982).

13. Thiringer, K.: Cord plasma hypoxanthine as a measure of foetal asphyxia. Comparison with clinical assessment and laboratory measures of asphyxia in newborn infants. Acta Paediatr. Scand., 72: 231 (1983).

14. Thiringer, K., Blomstrand, S., Hrbek, A., Karlsson, K., and Kjellmer, I. Cerebral arterio-venous difference for hypoxanthine and lactate during graded asphyxia in the fetal lamb. Brain Res., 239: 107 (1982).

15. Thiringer, K., Karlsson, K., and Rosén, K.-G.: Changes in hypoxanthine and lactate during and after hypoxia in the fetal sheep with chronically-implanted vascular catheters. J. Dev. Physiol., 3: 375 (1981).

16. Thiringer, K., Karlsson, K., Rosén, K.-G., and Kjellmer, I.: The contribution of heart muscle, liver, skeletal muscle and placenta to the elevation of hypoxanthine during asphyxia in the acutely exteriorized fetal lamb. Biol. Neonate (in press).

17. Thiringer, K., Saugstad, O. D., and Kjellmer, I.: Plama hypoxanthine in exteriorized, acutely asphyxiated fetal lambs. Pediatr. Res., 14: 905 (1980).

18. Tuchschmid, P. E., Boutellier, U., Koller, E. A., and Duc, G. V.: Comparison of hypoxanthine, lactate, and ECG signs as indicators of hypoxia. Pediatr. Res., 15: 28 (1981).

19. Requests for reprints should be addressed to: Dr. Kari O. Raivio, Children's Hospital, University of Helsinki, SF-00290 Helsinki 29, Finland.

20. This research was supported by The Sigrid Jusélius Foundation and the Foundation for Pediatric Research in Finland.

21. Received for publication February 17, 1983.

22. Accepted for publication July 7,1983 . 